\title{
My Approach to Assessing Right Ventricular Function After Tetralogy of Fallot Repair
}

\author{
Como Eu Faço a Avaliação do Ventrículo Direito no Pós-Operatório de Tetralogia de Fallot
}

\section{Laura Mercer-Rosa}

University of Pennsylvania Perelman School of Medicine; Director, Echolab Research Unit; Co-Director, Fellowship Program Children's Hospital Filadelfia, PA, USA

The assessment of right ventricular (RV) function in patients after surgical repair for tetralogy of Fallot (TOF) remains challenging. Over the many years of my career as an echocardiographer, I learned to trust my eyes when inspecting the RV before I moved on to quantitative parameters. Or as the saying goes: "The devil knows more because he is old than because he is the devil!" Here I will give a brief overview of the tools I use to best assess RV function after TOF repair. It is worth mentioning that following the recovery of function in the early post-operative period, RV systolic function is generally preserved in the first two decades of life when measured by cardiac magnetic resonance imaging, which remains the gold standard modality for assessing ejection fraction. ${ }^{1,2}$ With this in mind, using more sensitive quantification parameters is important, as subtle changes in function may not be apparent on visual inspection or using global parameters (ejection fraction). Finally, I emphasize that the "tipping" point of the $\mathrm{RV}$ when the it transitions from a state of preserved function to established dysfunction is unknown and constitutes a greatest gap in TOF-related knowledge. Hence, sensitive quantification methods are needed for the follow-up of these patients.

\section{Qualitative assessment}

On the apical four-chamber view, I examine the motion of the tricuspid valve toward the apex (measured as the tricuspid annular plane systolic excursion [TAPSE] as detailed below) and the motion of the RV wall toward the septum versus data of prior studies. Likewise, parasternal long- and short-axis views are important. In those with favorable acoustic windows, RV wall shortening can be assessed using M-mode (Figure 1). It is worth noting that the qualitative assessment of RV function should not be the sole parameter. ${ }^{3,4}$

\section{Quantitative assessment}

Multiple non-invasive measures are used to evaluate RV systolic function, including tricuspid annular plane systolic excursion (TAPSE), tissue Doppler-derived tricuspid systolic velocity $\left(S^{\prime}\right)$, fractional area change (FAC), longitudinal peak

\section{Keywords}

Tetralogy of Fallot; Ventricular Function; Resonance, Magnetic.

Mailing Address: Laura Mercer-Rosa •

E-mail: mercerrosal@email.chop.edu

Manuscript received 11/18/2021; revised 11/19/2021; accepted 11/25/2021.

DOI: $10.47593 / 2675-312 X / 20213404$ ecom 17 systolic strain, stress echocardiography, and three-dimensional echocardiography., 5-8

a. TAPSE and S' should only be used in the follow-up of TOF patients because a significant change in the longitudinal contraction of the RV occurs after TOF repair; therefore, low TAPSE and S' values do not indicate global RV dysfunction. ${ }^{6}$ However, these indices can be used longitudinally to detect changes in longitudinal shortening.

b. RV fractional area change (FAC) is superior to TAPSE and $\mathrm{S}^{\prime}$ because it measures change in area and, therefore, considers the contribution of the RV to the overall RV shortening. The FAC is ideal when the endocardial border is well visualized, which allows for precise tracing. This can be done at the time of the study, which is an additional advantage. ${ }^{8}$ First, the area of change is traced along the RV endocardium during diastole and systole. Second, the RV diastolic area is subtracted by the RV systolic area and then divided by the RV diastolic area to produce the FAC calculation (Figure 2). This is a relatively easy and reproducible parameter that has at least a modest correlation with cardiac magnetic resonance imaging data. ${ }^{3,9}$ Measurement of the RV FAC should be utilized more often, especially given the limitations of TAPSE and $S^{\prime}$ in patients with TOF.

c. Myocardial deformation: I am a proponent of the wider use of RV global longitudinal strain and RV free wall strain as measures of myocardial contractility. Strain calculates cardiac deformation as a percentage change in length. ${ }^{10}$ It is quantitative, angle-independent, and less dependent on loading conditions than other echocardiographic measures, which is a particularly convenient characteristic when managing a volume-loaded RV in patients with TOF. ${ }^{11,5,12}$ After training personnel and achieving adequate reproducibility, global longitudinal RV strain appears to be a sensitive measure of ventricular function, as evidence suggests that strain is affected prior to changes in other more blunt measures of function. ${ }^{13}$ Therefore, strain is a very important parameter in RV functional assessments after TOF repair. Newer cardiac ultrasound machines have RV strain built in and take measurements in a few seconds, with quite good tracking of the RV wall that requires minimal adjustment (Figure 3). I propose that it be added to the echocardiography report for all patients after TOF repair. ${ }^{12,14}$

In summary, when assessing the RV, I consider every available parameter. I also ensure that certain parameters are similar to confirm adequate measurement accuracy. For example, if the TAPSE is diminished, so is the tricuspid tissue velocity in systole $\left(\mathrm{S}^{\prime}\right)$. Likewise, when the TAPSE is diminished but the RV wall has better shortening, this is reflected in the FAC measurement, in which the contribution of the RV wall 


\section{Editorial}
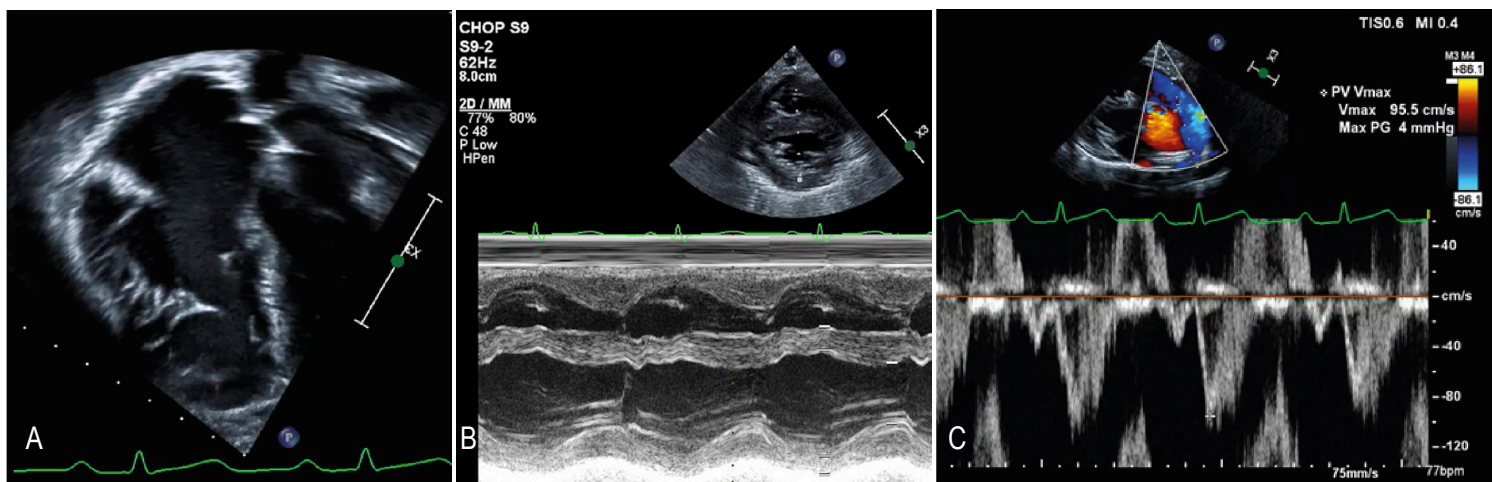

Figure 1 - (A) Demonstration of a dilated right ventricle after tetralogy of Fallot repair. (B) M-mode image demonstrating the shortening of the right ventricular (RV) anterior wall. Despite the significant $R V$ dilation, the wall shortens significantly. This modality complements the qualitative assessment and is quite useful in adequate acoustic windows. (C) Antegrade end-diastolic flow is visible in the main pulmonary artery in the presence of severe pulmonary insufficiency. This phenomenon is referred to as "restrictive physiology of the right ventricle" and indicates diastolic dysfunction. This is only seen in patients with greater than moderate pulmonary insufficiency.

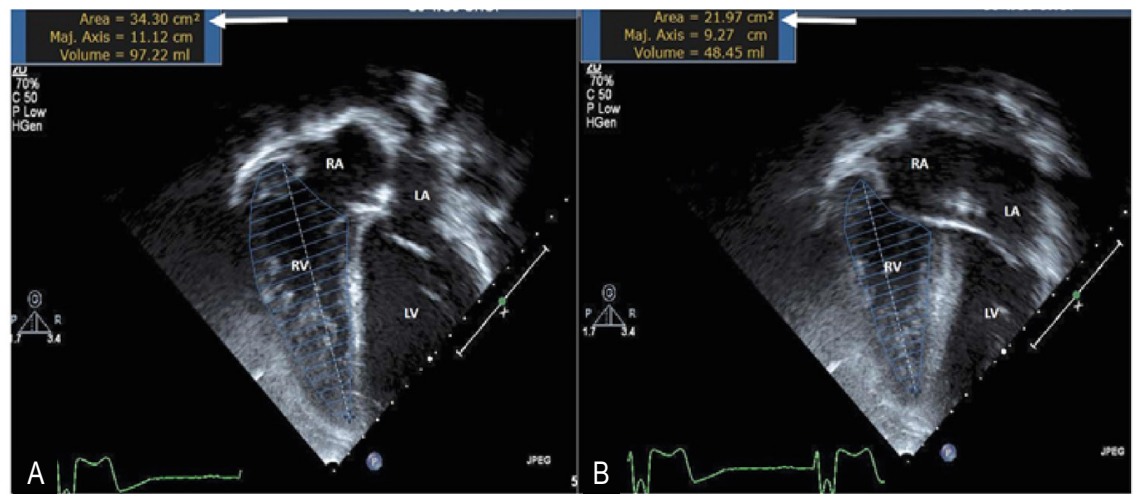

$F A C$, fractional area change; $L A$, left atrium; $L V$, left ventricle; $R A$, right atrium; $R V$, right ventricle.

Figure 2 - Calculation of fractional area change: Fractional area change measurement obtained during end-diastole $(A)$ and end-systole (B) in the right ventricle of a patient with tetralogy of Fallot. The arrows demonstrate the measured areas during diastole and systole. The calculated FAC [(34.3-21.97)/34.3] = 35.9\%, which is within normal limits.

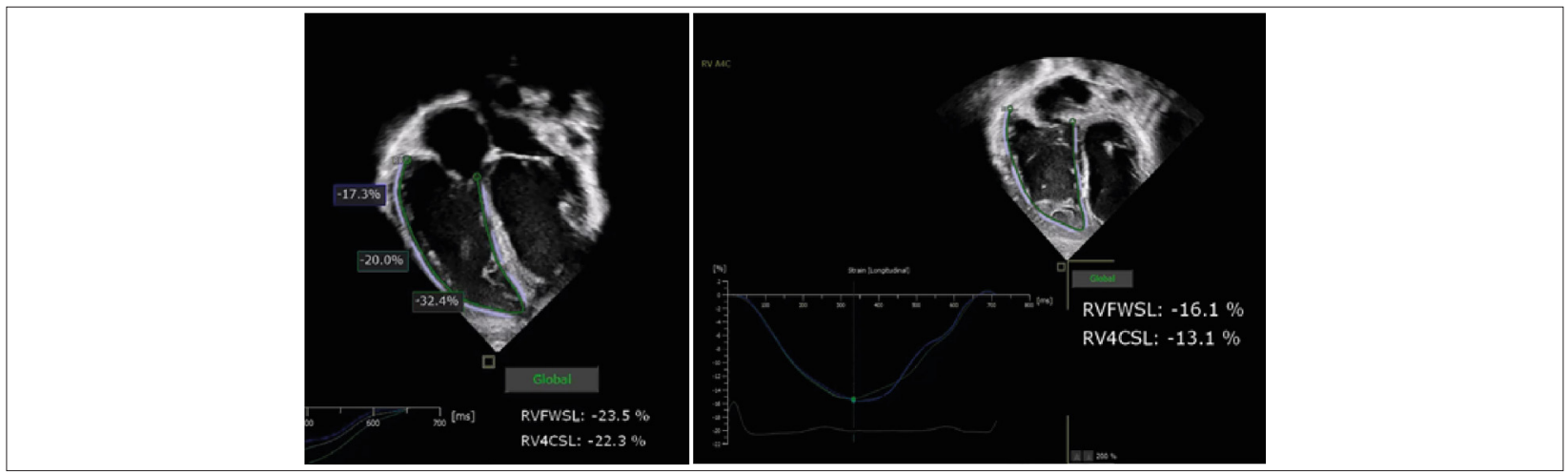

Figure 3-Real-time measurement of right ventricular (RV) global longitudinal strain and $R V$ free wall strain. The figure on the left demonstrates a dilated $R V$ with preserved $R V$ strain. The figure on the right demonstrates a dilated $R V$ and diminished $R V$ strain. Observe that the $R V$ free wall strain is better than the global strain. Some suggest limiting the use of strain to the RV free wall strain only, as opposed to the RV global longitudinal strain (which incorporates the septum). 


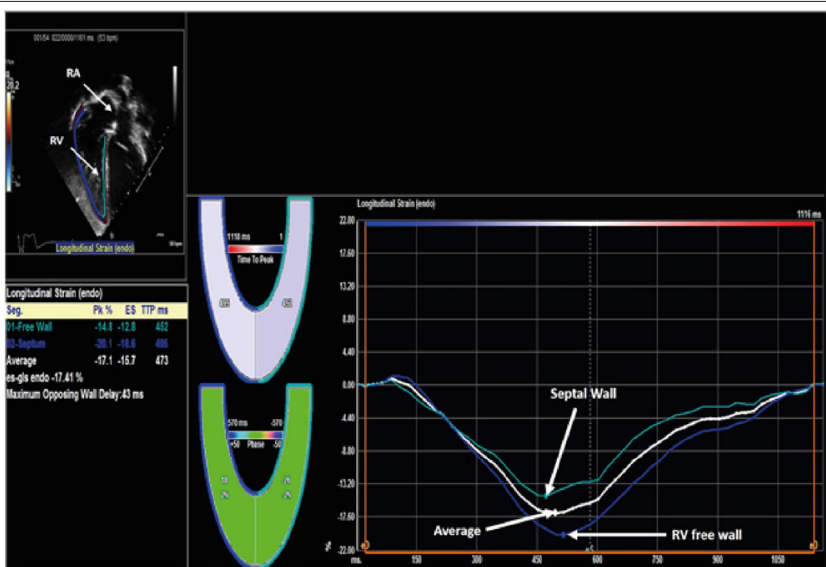

Figura 4 - Right ventricular global longitudinal strain (TomTecl). The curves demonstrate segmental wall motion of the right ventricular septum and free walls. RA, right atrium; $R V$, right ventricle.

to overall shortening is easily identified. Strain is very useful for identifying moderate versus mild or severe versus moderate dysfunction. Our eyes are usually able to detect when RV function is normal or severely diminished, but the nuances in between are quite challenging. As physicians, we strive to improve TOF patient outcomes. Thus, our collective goal should be to better understand RV mechanics, apply the best imaging tools to accurately measure RV function, identify patients at increased

\section{References}

1. Bhat M, Goldmuntz E, Fogel MA, RychikJ and Mercer-Rosa L. Longitudinal Validation of the Diastolic to Systolic Time-Velocity Integral Ratio as a Doppler-Derived Measure of Pulmonary Regurgitation in Patients with Repaired Tetralogy of Fallot. Pediatr Cardiol. 2017;38:240-6.

2. Bhat M, Mercer-Rosa L, Fogel MA, Harris MA, Paridon SM, McBride MG, Shults J, Zhang X and Goldmuntz E. Longitudinal changes in adolescents with TOF: implications for care. Eur Heart J Cardiovasc Imaging. 2017; 18:356-63.

3. Valente AM, Cook S, Festa P, Ko HH, Krishnamurthy R, Taylor AM, Warnes CA Kreutzer J and Geva T. Multimodality imaging guidelines for patients with repaired tetralogy of fallot: a report from the AmericanSsociety of Echocardiography: developed in collaboration with the Society for Cardiovascular Magnetic Resonance and the Society for Pediatric Radiology. J Am Soc Echocardiogr. 2014;27:111-41.

4. DiLorenzo MP, Bhatt SM and Mercer-Rosa L. How best to assess right ventricular function by echocardiography. Cardiol Young. 2015;25:1473-81.

5. DiLorenzo MP, Elci OU, Wang Y, Banerjee A, Sato T, Ky B, Goldmuntz E, etal. Longitudinal Changes in Right Ventricular Function in Tetralogy of Fallot in the Initial Years after Surgical Repair. J Am Soc Echocardiogr. 2018;31:816-21.

6. Mercer-Rosa L, Parnell A, Forfia PR, Yang W, Goldmuntz E and Kawut SM. Tricuspid annular plane systolic excursion in the assessment of right ventricular function in children and adolescents after repair of tetralogy of Fallot. J Am Soc Echocardiogr. 2013;26:1322-9.

7. Mercer-Rosa L, Yang W, Kutty S, RychikJ, Fogel M, Goldmuntz E. Quantifying pulmonary regurgitation and right ventricular function in surgically repaired tetralogy of Fallot: a comparative analysis of echocardiography and magnetic risk who may require more frequent monitoring or earlier intervention, predict clinical outcomes, and ultimately provide scientific evidence that guides patient care and treatment.

\section{Conflict of interest}

The author declared that have no conflict of interest.

resonance imaging. Circ Cardiovasc Imaging. 2012;5:637-43

8. White BR, Katcoff H, Faerber JA, Lin KY, Rossano JW, Mercer-Rosa L, et al Echocardiographic Assessment of Right Ventricular Function in Clinically Well Pediatric Heart Transplantation Patients and Comparison With Normal Control Subjects. J Am Soc Echocardiogr. 2019;32:537-544 e3.

9. Selly JB, Iriart X, Roubertie F, Mauriat P, MarekJ, Guilhon E, et al. Multivariable assessment of the right ventricle by echocardiography in patients with repaired tetralogy of Fallot undergoing pulmonary valve replacement: a comparative study with magnetic resonance imaging. Arch Cardiovasc Dis. 2015;108:5-15

10. Mertens LL, Friedberg MK. Imaging the right ventricle--current state of the art. Nat Rev Cardiol. 2010;7:551-63.

11. Toro KD, Soriano BD,Buddhe S. Right ventricular global longitudinal strain in repaired tetralogy of Fallot. Echocardiography. 2016;33:1557-1562.

12. Savla JJ, De Matteo V, Wang Y and Mercer-Rosa L. What Echocardiographic Measure Should Be Used to Assess Right Ventricular Function in Tetralogy of Fallot? J Cardiovasc Dis Diagn. 2017;5.

13. Mondillo S, Galderisi M, Mele D, Cameli M, Lomoriello VS, Zaca V, et al. Echocardiography Study Group Of The Italian Society Of C. Speckletracking echocardiography: a new technique for assessing myocardial function. J Ultrasound Med. 2011;30:71-83.

14. Scherptong RW, Mollema SA, Blom NA, Kroft LJ, de Roos A, Vliegen HW, et al. Right ventricular peak systolic longitudinal strain is a sensitive marker for right ventricular deterioration in adult patients with tetralogy of Fallot. Int J Cardiovasc Imaging. 2009;25:669-76. 